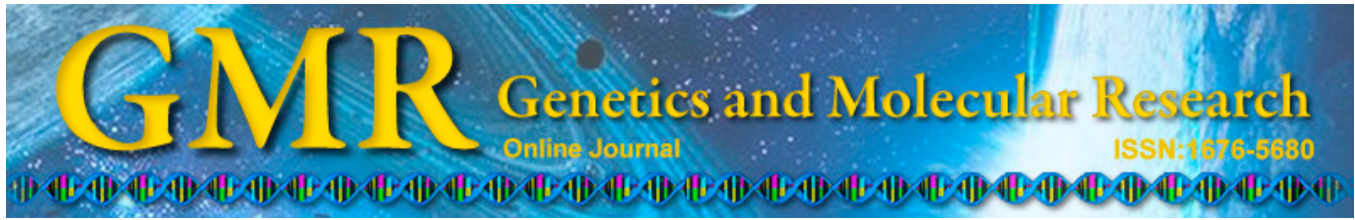

\title{
Expression and hypoxia adaptation analysis of the $E P O$ gene in different tissues of plateau Tibetan pigs
}

\section{B.Z. Deji ${ }^{1}$, P. Shang1, W.J. Danzeng ${ }^{1}$, H. Zhang² and Y.Z. Qiangba' ${ }^{1}$}

${ }^{1}$ Tibet Animal Genetics and Breeding Laboratory, College of Animal Science, Tibet Agriculture and Animal Husbandry University, Linzhi, China

${ }^{2}$ College of Animal Science and Technology, China Agricultural University, Beijing, China

Corresponding author: Y.Z. Qiangba

E-mail: qbyz628@yeah.net

Genet. Mol. Res. 14 (1): 1700-1706 (2015)

Received February 4, 2014

Accepted November 5, 2014

Published March 6, 2015

DOI http://dx.doi.org/10.4238/2015.March.6.16

\begin{abstract}
This study aimed to observe the expression characteristics of the erythropoietin $(E P O)$ gene in different tissues of Tibetan pigs and to explore the adaptation to hypoxic environments. The cDNA in heart, liver, lung, kidney, muscle, brain, and fat of Tibetan pigs was used as the template. Through the number of cycles of the polymerase chain reaction (PCR), annealing temperature, and system optimization, a stable and specific semi-quantitative PCR system was established. The $E P O$ gene in different tissues of Tibetan pigs was detected using this system. The results showed that the $E P O$ gene was expressed in heart, liver, lung, kidney, muscle, brain, and fat of the pigs. There were obvious differences in the expression in each tissue, and the expression sequence was as follows: kidney $>$ muscle $>$ lung $>$ brain $>$ liver $>$ heart $>$ fat. The results showed that the EPO gene was expressed in various tissues of Tibetan pigs. There were obvious differences in
\end{abstract}


expression and each tissue may play a different regulatory role in the adaptation to hypoxia.

Key words: Tibetan pig; Erythropoietin; Gene expression; Hypoxic adaptation

\section{INTRODUCTION}

Erythropoietin (EPO) was the first discovered and clinically used hematopoietic growth factor. As early as 1906, Carnot et al. found that after blood loss in rabbit, the body can produce an effect on the hematopoietic system to accelerate the levels of EPO in the peripheral blood (Jelkmann, 2007). Thus, they proposed the existence of a humoral factor that could regulate blood cell production in a feedback manner. Thirty years later, this view was confirmed, and this factor was named EPO. In 1957, Jacobson et al. found that the kidney was the main organ producing EPO. Liver, macrophages, and nucleated red blood cells can also produce EPO. However, EPO production outside of the kidneys produces less than 10 to $15 \%$ of the total amount. Fetuses and newborns largely rely on the liver for EPO production (Brace et al., 2006). In recent years, it was discovered that brain astrocytes can produce EPO. Experiments confirmed that EPO produced by the brain tissue can protect neurons from ischemic injury (Sakanaka et al., 1998). Hermine et al. (1991) and Stopka et al. (1998) found that erythroid progenitor cells can also express a small amount of EPO. This indicated that low levels of EPO were regulated in an autocrine manner. The circulating EPO could stimulate the bone marrow to produce large amounts of red blood cells under hypoxia and other stimuli. Numerous studies confirmed that EPO acted on the target tissue and target cells through autocrine, paracrine, and endocrine manners to perform a variety of biological functions.

According to information from the National Center for Biotechnology Information (NCBI), the pig EPO gene is located on chromosome 3, and it consists of 4 introns and 5 exons. The genomic DNA length was $2429 \mathrm{bp}$, the mRNA sequence length was $1263 \mathrm{bp}$, the length of the coding region was 120-704 bp in the whole genome sequences, and it encodes 194 amino acids. The main biological functions of EPO include anti-apoptotic effects, anti-inflammatory effects, antioxidant effects, angiogenesis-promoting effects, effects on the immune system, erythropoiesis-regulating effects, mitogenic effects, chemotactic effects, angiogenesis effects, and intracellular calcium mobilization effects. Altitude sickness can be used to indicate genes and related molecular pathways that are associated with hypoxia adaptation. Some genes associated with hypoxia adaptation were found, such as $E P O$, erythropoietin receptor $(E P O R)$, angiotensin-converting enzyme ( $A C E)$, aldosterone synthase (CYP11B2), and NO metabolismassociated genes (NOS1, NOS2, and NOS3). The relationship between the generation of EPO and the oxygen status of the body had already been confirmed, and the mechanism is now under study. In hypoxia (anemia, hypovolemia) and other states, kidneys will secrete large amounts of EPO into the bloodstream. When the blood circulation reaches the bone marrow, it acts on the hematopoietic stem cells to stimulate the proliferation of red blood cells until the amount of oxygen carried by red blood cells meets the body's normal level. Currently, there are many of the studies on the $E P O$ gene, but $E P O$ gene expression studies in Tibetan pigs are rare.

Oxygen is a necessary environmental factor for biological growth and survival. Hypoxia is a significant environmental pressure for biological survival. For humans and other terrestrial vertebrates, hypoxic stress can lead to many metabolic changes to achieve fast 
adaptation. Tibetan pig is a mammal that has strong resistance to hypoxia. However, differences in the response to hypoxia in different studies resulted in a lot of misunderstanding of the molecular mechanisms of hypoxia resistance in Tibetan pigs. Under normal oxygen, the kidney only expresses small amounts of EPO mRNA, indicating that the kidney had no reserve EPO (Jelkmann and Bauer, 1981). One hour after hypoxia, EPO mRNA levels began to increase, serum EPO levels were elevated 1.5-2 h after hypoxia, and, after 6-24 h of hypoxia, EPO expression reached its peak in rodents. It reached a peak within $48 \mathrm{~h}$ and then began to decline. In this study, we investigated the mRNA expression of the EPO gene in Tibetan pigs, and we also studied the expression differences in different tissues to further explore the expression regulation mechanism of the $E P O$ gene in multiple tissues of Tibetan pigs experiencing hypoxia to lay the foundation for future analysis and to identify new ways to respond to diseases. Of the many approaches that are used to challenge disease, candidate gene selection for disease resistance to improve the overall immunity of pigs may be the most effective approach.

\section{MATERIAL AND METHODS}

\section{Material}

This trial randomly selected 6-month-old, healthy Tibetan pigs that were provided by Tibet pig farms in ZhongSa Village, GongBuDa County, LinZhi Prefecture, Tibet. The heart, liver, lung, kidney, muscle, brain, fat, and other tissues were collected as the experimental materials. The collected tissues were immersed and frozen in liquid nitrogen and stored at $-80^{\circ} \mathrm{C}$ for polymerase chain reaction (PCR) of the $E P O$ gene.

\section{PCR primer design}

From the NCBI database, the mRNA sequences of pig EPO and $\beta$-actin were downloaded, and the Premier 5.0 software was used to design the semi-quantitative primers (Table 1). Primers were synthesized by Beijing Liuhe Synthetic Genomics Inc., and they were water dissolved in $0.1 \%$ diethylpyrocarbonate (DEPC) and preserved at $4^{\circ} \mathrm{C}$.

Table 1. Primers used in amplification.

\begin{tabular}{llc}
\hline Primer name & Sequence & Product length (bp) \\
\hline EPO-F & 5'-GCAGGAGGAATTCAGAGATCA-3' & 183 \\
EPO-R & 5'-AATTCATGGCTTGCCTCCTA-3' & 127 \\
ACT-F & 5'-TCTGGCACCACACCTTCTA-3' & \\
ACT-R & 5'-AAGGTCTCGAACATGATCTG-3' & \\
\hline
\end{tabular}

\section{Extraction of total RNA, purity detection, and quantification}

Total RNA extraction was performed using a kit according to manufacturer instructions.

\section{Electrophoresis}

Total RNA was extracted with TRIZOL according to the test procedure to perform 
quantitative and qualitative detection (electrophoresis). The RNA integrity was determined with 7\% agarose gel electrophoresis, and the final RNA did not show significant degradation phenomena; $28 \mathrm{~S}, 18 \mathrm{~S}$, and $5 \mathrm{~S}$ bands were clear and visible without smearing. The concentration ratio of the $28 \mathrm{~S}$ and $18 \mathrm{~S}$ bands was close to $2: 1$, and RNA with the best integrity was selected for the following experiments.

\section{Ultraviolet spectrophotometer detection}

The final RNA precipitation was dissolved in an appropriate amount of DEPC water. A Nanodrop 2000 (Thermo Scientific, Waltham, MA, USA) spectrophotometer was used to determine the concentration, the optical density at $260 \mathrm{~nm}\left(\mathrm{OD}_{260}\right) / \mathrm{OD}_{280}$ was calculated, and the total RNA purity was estimated.

\section{cDNA synthesis}

An M-MLV reverse transcription kit (Beijing QuanShiJing Biotechnology Co., Ltd., China) was used to synthesize cDNA, which was stored at $-20^{\circ} \mathrm{C}$. cDNA was synthesized at $45^{\circ} \mathrm{C}$ for $30 \mathrm{~min}$ and the reaction was terminated at $85^{\circ} \mathrm{C}$ for $5 \mathrm{~min}$. The reverse transcription product of the housekeeping $\beta$-actin gene was tested by ordinary PCR amplification (Table 2).

Table 2. Pig EPO gene RNA reverse transcription reaction system.
\begin{tabular}{lcc}
\hline Reagents & Concentration & Volume $(\mu \mathrm{L})$ \\
\hline RNA & $1 \mu \mathrm{g} / \mu \mathrm{L}$ & 3 \\
Premer Oligo (dT)18 & $10 \mathrm{pmol} / \mu \mathrm{L}$ & 1 \\
RNase-free ddH $2 \mathrm{O}$ & & 5 \\
$2 \mathrm{X}$ ES & & 10 \\
ESMix & & 1 \\
\hline
\end{tabular}

\section{RESULTS}

\section{Total RNA integrity and purity testing in tissues of Tibetan pigs}

The RNA from heart, liver, lung, kidney, muscle, brain, and fat of Tibetan pigs was extracted using the TRIZOL method, $30-50 \mu \mathrm{L} 1 \%$ DEPC water was added, and $1 \mu \mathrm{L}$ was used for $1 \%$ agarose gel electrophoresis. The electrophoresis showed that the integrity was good (Figure 1). The $\mathrm{OD}_{260} / \mathrm{OD}_{280}$ values were greater than 1.8 to 2.0, indicating that it can be used for PCR. The samples did not have protein and DNA contamination, and the quality of the total RNA was good and could be used for subsequent cDNA synthesis and semi-quantitative analysis.

\section{Semi-quantitative PCR analysis of the EPO gene in different tissues of Tibetan pigs}

Using an established PCR system and adjusting the cDNA template amount, the internal control gene $\beta$-actin was amplified, and the brightness of the PCR product bands was made to be consistent in the different tissues in Tibetan pigs. The $\beta$-actin adjustment system was 
used to amplify the target gene of $E P O$. The test was repeated 3 times. The relative expression of the $E P O$ gene was calculated, and the results are shown in Figure 2. The EPO gene was expressed in heart, liver, lung, kidney, muscle, brain, and fat tissues. The expression levels varied in different tissues. The highest expression was in the kidney, followed by heart, muscle, lung, liver, fat, and brain. The band from the brain showed the weakest brightness.

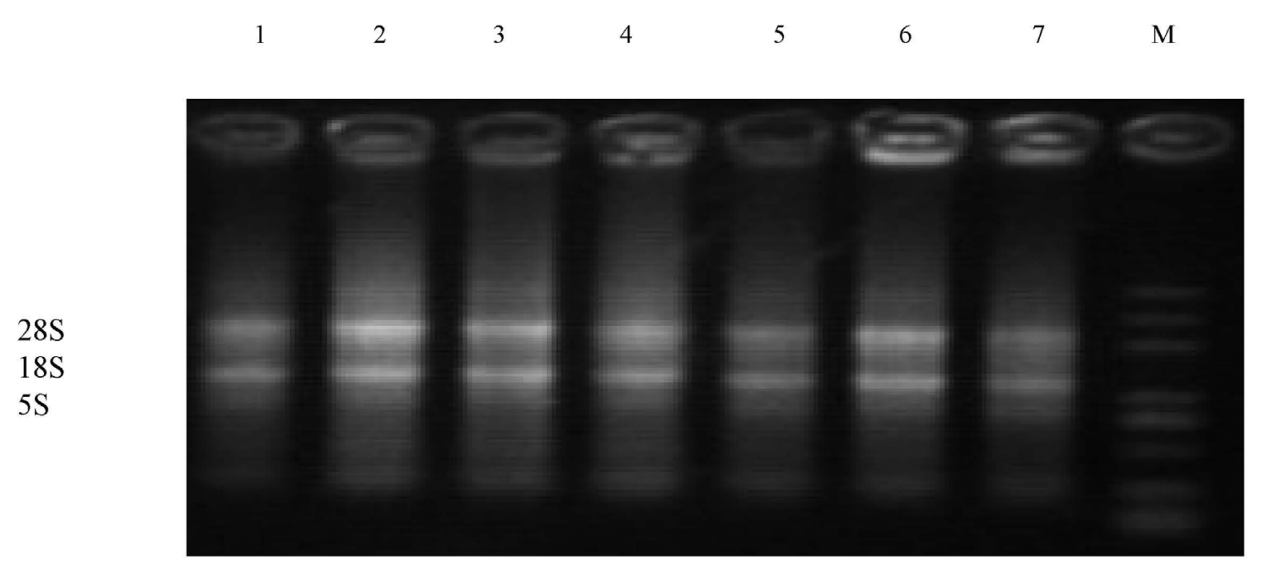

Figure 1. Total RNA electrophoresis detection. Lanes 1-7 = heart, liver, lung, kidney, muscle, brain, and fat, respectively; lane $M=\mathrm{DM} 2000$ Plus marker.

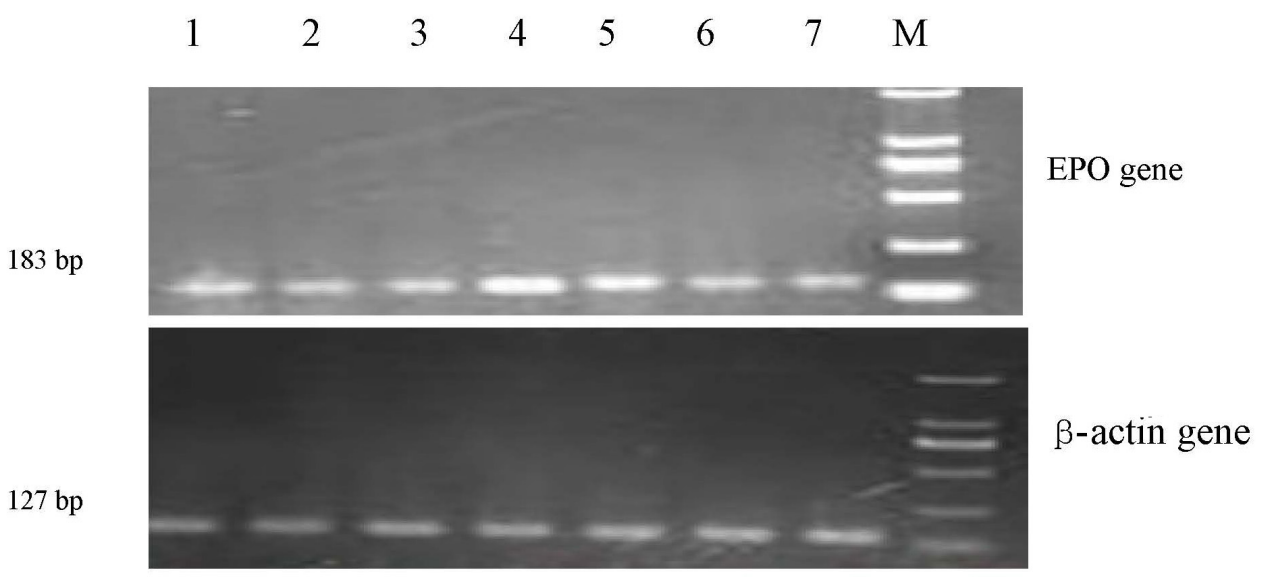

Figure 2. Semi-quantitative polymerase chain reaction to detect $E P O$ genes differentially expressed in different tissues in Tibetan pig. Lanes 1-7 = heart, liver, lung, kidney, muscle, brain, and fat, respectively; lane $M=\mathrm{DM}$ 2000 Plus marker.

\section{DISCUSSION}

PCR is a rapid DNA amplification technique and a means of genetic testing. It has high sensitivity, specificity, and fast and easy features. When gene expression is rare in organisms, traditional detection methods can hardly detect it. However, using PCR, the DNA 
fragments can be amplified one million times or more, allowing the gene expression to be quantified. During the PCR process, PCR products increased by a cumulative index when the cycles began, and the products and the template showed a linear relationship. Through PCR products, we can compare target gene expression in total RNA (Ding et al., 2012). After a certain number of cycles, PCR products no longer accumulate exponentially, and a plateau phase is entered. Therefore, to avoid these effects, an appropriate number of cycles is one of the key factors in semi-quantitative PCR.

In this experiment, through a series of PCR amplification cycles of the EPO target gene and the reference gene $\beta$-actin, the exponential growth phase of 2 pairs of primers and the number of amplification cycles of the 2 genes were determined. To avoid quality differences and amplification efficiency differences between sample templates, an internal control system was introduced in the PCR. The commonly used reference genes are $\beta$-actin and glyceraldehyde3 -phosphate dehydrogenase. Because the transcription of these genes was relatively stable, the relative content was rich. This experiment used the $\beta$-actin gene as the reference gene; it was amplified from Tibetan pigs from different organizations. The results showed that the expression was relatively stable and could be used as an internal control in detecting target gene expression. Hypoxia (or plateau stress) was the main reason for EPO secretion. Secretion was mainly from tubulointerstitial cells and liver parenchymal cells and was the body's essential response to hypoxia. In the hypoxic environment, the tissue cells of Tibetan pigs may receive hypoxia signals through oxygen acceptor molecules on their membrane. The EPO gene in hypoxic response has complex responses. In summary, hypoxia caused the hypoxic response, which included $E P O$ gene expression and regulation changes in Tibetan pigs. The EPO gene was involved in hypoxic sensing of the internal environment and hypoxia signal transduction. The hypoxia adaptation of Tibetan pigs may be an important direction for future research.

In recent years, many studies have shown that the $E P O$ gene was expressed in different tissues of animals, such as the central nervous system, female reproductive system, and solid tumor tissue (Cai et al., 2004). EPO is traditionally considered as a type of blood cell factor. However, in recent years, many studies have shown that the $E P O$ gene is widely expressed in embryonic and adult tissues, but the expression levels were low in body fluids.

In this experiment, we built a stable and specific semi-quantitative standard PCR system to detect the tissue-specific expression of the EPO gene in Tibetan pigs. The results showed that the gene was expressed in the heart, liver, lung, kidney, muscle, brain, and fat. The strongest expression was in the kidney, muscle, lung, and brain, followed by the heart and liver, and expression was the weakest in fat. During hypoxia (anemia, hypovolemia), the kidney is the main organ secreting and transporting EPO in animals. The kidney experienced the oxygen concentration changes through the corresponding cells (renal cortex cells); then, it regulated the production of EPO to participate in the process of erythropoiesis. Once the body showed the hypoxia (caused by the anemia or hypovolemia), the kidney will secrete large amounts of EPO into the bloodstream, and EPO reaches the bone marrow with the blood circulation and acts on hematopoietic stem cells to promote red blood cell hyperplasia until the amount of oxygen carried by red blood cells reaches the body's normal levels. Fan and Lin (2009) found that EPO and EPOR normal brain tissue showed low expression; hypoxia, ischemia, and other pathological states increased expression. EPO concentrations in cerebrospinal fluid significantly increased. The heart is the dynamic organ in blood circulation. Anoxia is a source of stress that can cause sympathetic excitement; the sympathetic-adrenal axis activity increased, thereby increasing myocardial contractility and heart rate (Fu et al., 2007). 
Long-term hypoxia made adaptive changes in myocardial morphology, structure, and function to adapt to the hypoxic environment (Martinez-Bello et al., 2011). EPO and EPOR expression was regulated by tissue oxygenation. When the oxygen supply was deficient in tissues, $E P O$ and $E P O R$ expression increased in the brain, kidneys, and liver. The hypoxia-dependent expression increase of $E P O$ and $E P O R$ was regulated mainly by hypoxia-inducible factor-1 (Heidbreder et al., 2003). The EPO gene played an important role in mediating and transporting in hypoxia and hypothermia. EPO gene expression patterns were different in different tissues of plateau animals. This indicated that different tissues of plateau animals had different responses to hypoxia and that it was an adaptive mechanism to hypoxia in different tissues of plateau animals.

This study showed that the EPO gene was expressed in all tissues in Tibetan pigs, especially in the kidney, which showed tissue-specific features. The EPO gene had the highest expression in the kidney of various tissues of pigs. This indicated that after hypoxia, the kidney played an important role in the EPO production. Furthermore, the possible physiological function of the EPO gene in other parts of the pig was also worth exploring. In the same tissues of different species, the expression regulation and physiological function of the EPO gene will be further studied.

\section{ACKNOWLEDGMENTS}

Research supported by the National Science and Technology Support Project (\#2012BAD03B03) and the National Natural Science Foundation of China (\#31040077).

\section{REFERENCES}

Brace RA, Cheung CY, Davis LE, Gagnon R, et al. (2006). Sources of amniotic fluid erythropoietin during normoxia and hypoxia in fetal sheep. Am. J. Obstet. Gynecol. 195: 246-254.

Cai GQ, Chen B, Fan Y, Ma XD, et al. (2004). Detection of Epo and VEGF mRNA in endometriosis tissues using hybridization in situ. J. Fourth Military Med. Univ. 2004: 548-550.

Ding YL, Li HM, Lin YF, He SG, et al. (2012). Expression analysis of EgPIP1;3 gene in cut eustoma flowers by semiquantitative RT-PCR. J. Zhongkai Univ. Agric. Technol. 2012: 6-10.

Fan G and Lin LM (2009) Erythropoietin and cerebral ischemic injury. J. Baotou Med. Coll. 25: 116-118.

Fu Q, Townsend NE, Shiller SM, Martini ER, et al. (2007). Intermittent hypobaric hypoxia exposure does not cause sustained alterations in autonomic control of blood pressure in young athletes. Am. J. Physiol. Regul. Integr. Comp. Physiol. 292: R1977-R1984.

Heidbreder M, Fröhlich F, Jöhren O, Dendorfer A, et al. (2003). Hypoxia rapidly activates HIF-3alpha mRNA expression. FASEB J. 17: 1541-1543.

Hermine O, Beru N, Pech N and Goldwasser E (1991). An autocrine role for erythropoietin in mouse hematopoietic cell differentiation. Blood 78: 2253-2260.

Jacobson LO, Goldwasser E, Fried W and Plzak LF (1957). Studies on erythropoiesis. VII. The role of the kidney in the production of erythropoietin. Trans. Assoc. Am. Physicians 70: 305-317.

Jelkmann W (2007). Erythropoietin after a century of research: younger than ever. Eur. J. Haematol. 78: 183-205.

Jelkmann W and Bauer C (1981). Demonstration of high levels of erythropoietin in rat kidneys following hypoxic hypoxia. Pflugers Arch. 392: 34-39.

Martinez-Bello VE, Sanchis-Gomar F, Nascimento AL, Pallardo FV, et al. (2011). Living at high altitude in combination with sea-level sprint training increases hematological parameters but does not improve performance in rats. Eur. J. Appl. Physiol. 111: 1147-1156.

Sakanaka M, Wen TC, Matsuda S, Masuda S, et al. (1998). In vivo evidence that erythropoietin protects neurons from ischemic damage. Proc. Natl. Acad. Sci. U. S. A. 95: 4635-4640.

Stopka T, Zivny JH, Stopkova P, Prchal JF, et al. (1998). Human hematopoietic progenitors express erythropoietin. Blood 91: 3766-3772. 